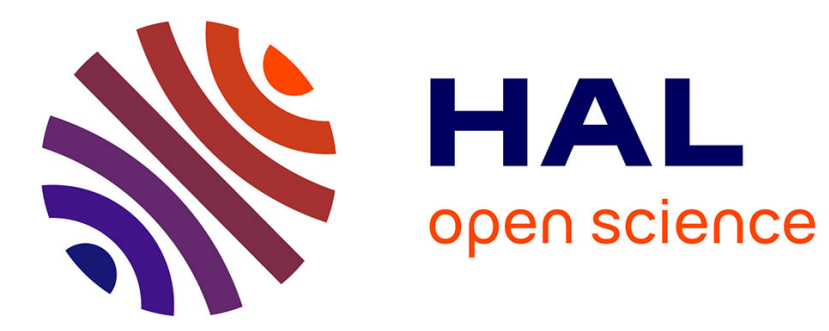

\title{
Psychologie et économie de marché
}

Evelyne Clément, Anne-Claude Juillerat van Der Linden, Guy Tiberghien, Martial van Der Linden, Bruno Vivicorsi

\section{To cite this version:}

Evelyne Clément, Anne-Claude Juillerat van Der Linden, Guy Tiberghien, Martial van Der Linden, Bruno Vivicorsi. Psychologie et économie de marché. Bulletin de psychologie, 2013, Numéro 527 (5), pp.437-440. 10.3917/bupsy.527.0437 . hal-02004925

\section{HAL Id: hal-02004925 https://hal-cyu.archives-ouvertes.fr/hal-02004925}

Submitted on 12 Feb 2019

HAL is a multi-disciplinary open access archive for the deposit and dissemination of scientific research documents, whether they are published or not. The documents may come from teaching and research institutions in France or abroad, or from public or private research centers.
L'archive ouverte pluridisciplinaire HAL, est destinée au dépôt et à la diffusion de documents scientifiques de niveau recherche, publiés ou non, émanant des établissements d'enseignement et de recherche français ou étrangers, des laboratoires publics ou privés. 


\title{
Psychologie et économie de marché
}

\author{
Clément Evelyne *
}

Juillerat Van der Linden Anne-Claude **

Tiberghien Guy ${ }^{* * *}$

Van der Linden Martial ${ }^{* *}$

Vivicorsi Bruno *

Résumé

Dans cet article, nous démontrons comment le discours pseudo-scientifique de « la preuve par le cerveau » et la dérive bio-médicale, visant à naturaliser l'esprit, participent à un système d'économie de marché qui réduit connaissances et individus à des marchandises comme les autres.

Psychology and Market Economy

Abstract

In this paper, we show how the pseudo-scientific discourse of «proof by the brain » and biomedical drift to naturalize the mind participate in a market economy that reduces knowledge and individuals to only goods.

Instrumentalisation de la psychologie scientifique ou discours pseudo-scientifique de « preuve par le cerveau », afin de naturaliser l'esprit, conception biomédicale et normative des conduites et du développement tout au long de la vie sont des fers de lance de l'idéologie libérale.

Que la psychologie, comme d'autres sciences humaines et sociales (voir, par exemple, Winkin, 2003), soit un instrument idéologique « des démocraties de marché », n’est pas

\footnotetext{
* Université de Rouen.

** Université de Genève.

*** Institut universitaire de France.

Correspondance : Évelyne Clément, Université de Rouen, Département de psychologie, 76821 Mont-SaintAignan Cedex,

$<$ evelyne.clement@univ-rouen.fr>
} 
nouveau. À titre d'exemple, en 1928, Edward Bernays, à partir des écrits de son oncle Sigmund Freud et des travaux sur la psychologie des foules, publiait, à l'usage des décideurs politiques et économiques américains, Propaganda, un petit guide pratique sur la manipulation mentale de l'opinion publique dans les sociétés démocratiques.

Aujourd'hui, c'est dans le contexte des politiques de l'Organisation mondiale du commerce (1995) sur l'accord général sur le commerce des services (AGCS), et des politiques européennes en matière d'économie de la connaissance (Lisbonne, 2000), que l'on peut s'interroger sur l'instrumentalisation de la psychologie pour justifier - prétendument scientifiquement - le libéralisme économique. En effet, suite à la Déclaration de Bologne, en 1999, les chefs d'États et de gouvernements, réunis en Conseil européen à Lisbonne, décident de faire de l'Union européenne « l'économie de la connaissance la plus compétitive et la plus dynamique du monde $[\ldots] »$.

Dans le contexte de l'AGCS, où l'éducation, l'enseignement supérieur, mais aussi la santé sont des services à privatiser comme les autres, il s'agit, entre autres, de normaliser et standardiser la connaissance, condition essentielle du processus de son échange et de sa circulation.

On voit là le rôle accordé à la psychologie par les démocraties de marché et la place qu'on veut lui donner dans la gestion des affaires humaines.

\section{PARADIGMES DE RECHERCHE EN PSYCHOLOGIE ET LOIS DU MARCHÉ}

L'intrication entre recherche et appareil de production, en fonction des progrès technologiques, n'est pas nouvelle et elle traverse le domaine de la psychologie scientifique depuis son avènement. Ce n'est pas un hasard si, dans la première moitié du $\mathrm{XX}^{\mathrm{e}}$ siècle, où l'économie manufacturière atteint son apogée aux États-Unis d'Amérique, le courant 
behavioriste s'impose en psychologie. Bien sûr, la psychologie behavioriste avait d'autres visées, proprement scientifiques. Il s'agissait d'établir des lois objectives régissant le comportement d'un individu dans un environnement physique, dans un contexte social, mais aussi au cours des apprentissages et du développement. Mais une des premières dérives de la psychologie comportementale appliquée a été de viser l'adaptation psychotechnologique et d'optimiser les rapports tayloriens de l'homme et de la machine. Le schéma S-R (stimulusréponse) révèle un évident isomorphisme avec le plan de la machine-outil : l'individu est une « machine » qui doit transformer les entrées sensorielles en comportements ajustés et adaptés à son environnement, comme une machine-outil transforme des matières premières en produits manufacturés, c'est-à-dire en marchandises.

Si la psychologie du comportement est inséparable de l'avènement de la société de production manufacturière, la psychologie de la cognition s'est développée, après la seconde guerre mondiale, en étroite relation avec l'essor de l'informatique fondamentale et de l'intelligence artificielle (IA), relation qui contribuera, en grande partie, à la naissance et l'essor initial des sciences cognitives. La production de biens manufacturés n'empêchant pas une diminution du taux de profit, un nouveau marché, aux implications stratégiques, militaires et économiques, était à conquérir : celui de l'information. L'information devient alors un moyen essentiel et continu d'innovation et de développement pour l'économie capitaliste. Dans ce contexte, la psychologie a été fortement modelée, dans un premier temps, par le marché technologique de l'informatique et de l'IA. Une grande partie des connaissances empiriques et théoriques de la psychologie cognitive a alors servi à justifier et/ou à mettre au point des systèmes ergonomiques d'optimisation de la production ou des systèmes logiciels experts (plus ou moins !) de reconnaissance automatique, d'apprentissage automatique, d'interfaces de communication, de fouille documentaire, de contrôle social, etc. 
Plus récemment, il y a une vingtaine d'années, c'est le nouveau marché en plein essor des techniques de neuro-imagerie qui a, peu à peu, façonné et contraint les recherches en psychologie. Ce dernier a ainsi permis aux neurosciences de s'imposer avec force dans les sciences cognitives et affectives, pour en disputer (ou en partager ?) le leadership avec l'IA. L'une des principales raisons tient aux sources de profits considérables qu'offre ce nouveau marché : la mesure de l'activité cérébrale nécessite en effet des systèmes informatiques et physiques complexes et coûteux (par exemple, le scanner IRM 3 teslas, acquis par le Commissariat à l'énergie atomique, pour son programme NeuroSpin, à Orsay, a coûté près de 51 millions d'euros et le coût d'un système IRM de base est de l'ordre, actuellement, de 2 millions d'euros). De tels systèmes exigent aussi un environnement très coûteux et des emplois très qualifiés (physiciens, neurophysiologistes, neuro-anatomistes, neurologues, informaticiens, psychologues). Les contraintes économiques de ce marché sont impitoyables, car il faut bien évidemment justifier de tels investissements de recherche. En quelques années, les pressions sont devenues particulièrement fortes pour que les recherches en psychologie se fondent, le plus souvent possible, sur des données d'imagerie cérébrale, même si celles-ci n'améliorent pas significativement l'explication des processus psychologiques, car « comprendre comment fonctionne chaque neurone et l'ensemble des neurones ne nous dit absolument rien de la façon dont le cerveau fabrique un état mental » (Gazzaniga, 2010, p. 291). Plus généralement, on assiste à une mise en question croissante de la légitimité des niveaux d'analyse psychologique et sociologique.

La psychologie est donc, de ce fait, devenue très dépendante des neurosciences sur le plan technologique, et de l'environnement médical et hospitalier sur le plan institutionnel. Mais elle est aussi devenue théoriquement dépendante des neurosciences dans le cadre de la stratégie, plus ou moins réductionniste, de naturalisation de l'esprit et de l'illusion entretenue que l'on peut lire l'esprit dans le cerveau (Tiberghien, 2011 ; Guillaume, Tiberghien, 
Baudouin, 2013). La preuve «par le cerveau » est devenue, ainsi, une quasi-nécessité pour publier ou répondre à de nombreux appels d'offres dans le domaine. Cela explique l'explosion des «neuro-quelque chose » (neurocognition, neuro-économie, neuromarketing, neuropédagogie, neuropolitique, neurophilosophie, etc.). Il n'y a, d'ailleurs, pas que l'esprit qui doit être naturalisé, le social doit l'être aussi (la morale, les émotions, la croyance religieuse, etc.).

Ainsi, le réductionnisme cérébral et, plus largement, neurobiologique, est devenu une pensée dominante (une neuroculture ; voir Williams, Higgs, Katz, 2012). Cette neuroculture conduit à réduire les comportements, les croyances et les compétences à leurs seuls déterminants biologiques et, comme l'indiquent Guillaume, Tiberghien et Baudouin (2013), elles font oublier que « notre vie psychique est aussi le produit de nos apprentissages, de notre trajectoire biographique, des caractéristiques socioculturelles et du milieu dans lequel on évolue ».

De plus en plus de psychologues répondent aux sirènes de la neuroculture et s'efforcent de localiser toutes ces réalités cognitives, affectives ou sociales dans le cerveau. Ce faisant, ils risquent fort de réduire la psychologie à n'être plus qu'une simple composante méthodologique et heuristique des neurosciences (Tiberghien, 2007). Si cela se confirmait, les chercheurs en psychologie deviendraient alors de simples sous-traitants des sciences biomédicales (neurosciences, neuropsychiatrie, neuropharmacologie). En définitive, le risque existe de voir, peu à peu, la recherche en psychologie absorbée par ce pôle à rentabilité économique élevée.

De telles implications sur le développement des recherches en psychologie ont aussi des conséquences dans le domaine social, professionnel et de la vie privée, où normalisation des comportements et conception biomédicale du développement humain répondent, là encore, à des logiques de marché. 


\section{LOIS DU MARCHÉ ET INTERVENTIONS PSYCHOLOGIQUES}

Les programmes très en vogue aujourd'hui de promotion de la santé mentale, mis en œuvre notamment dans le domaine de la prévention précoce des risques (de la grossesse à la vie adulte), visent à prédire et/ou modifier, le cas échéant, le comportement de l'individu pour, in fine, faire fructifier son capital humain. Les promoteurs de tels programmes s'appuient de la sorte sur certaines grilles de lecture ou d'évaluation psychologique pour en extraire des outils diagnostiques clé en main, très simples, prêts à l'emploi par tout (bon) professionnel sensible au développement (optimal) de l'enfant, à l'adaptabilité-flexibilité de l'individu face aux lois du marché et de la concurrence, au mieux-vieillir (non-vieillir ?) des personnes les plus âgées.

De façon plus spécifique, la neuro-culture dominante conduit à penser qu'il faut traiter, gérer, protéger, stimuler ou augmenter les capacités (cognitives) du cerveau et, dès lors, améliorer ou optimiser la condition humaine. Il s'agit d'accroître la santé cérébrale («neurobics »), tout comme l'activité physique («aerobics ») peut améliorer la santé pulmonaire et cardiovasculaire. On assiste en fait à une sorte de « fétichisation » du cerveau, lequel est perçu comme une entité privilégiée, distincte, que les individus sains doivent constamment stimuler, recâbler, reconstruire, nourrir, et dont ils doivent s'occuper s'ils veulent se maintenir mentalement en bonne santé et conserver leur identité.

Cela correspond bien aux principes du néo-libéralisme, qui attribuent une valeur primordiale au rendement, à l'efficacité, à la compétition et à l'individu, lequel est considéré comme ayant une propension intrinsèque à l'autosuffisance et à la protection de son individualité (sa vérité fondamentale), plutôt que des besoins plus vastes de la communauté. On assiste ainsi au développement de produits visant à améliorer le fonctionnement cérébral 
(stimulants cérébraux), de technologies d'optimisation ou de compensation cognitive (« neuro-prothèses ») ou d'objectifs sociaux (« neuro-diversité »).

Le risque de transformer le savoir scientifique en dispositif d'asservissement social (Gori, 2013) est grand : réduction de la complexité des comportements humains pour établir et imposer des normes édictées en règles de conduite (à tenir ou à soigner), normes fondées sur un modèle biomédical du comportement plus propice à supporter un projet politique de prédiction des comportements et de contrôle des populations (Parazelli, 2011).

Cette dérive biomédicale - et les réponses pharmacothérapeutiques qui en découlent - s'est imposée dans le diagnostic des troubles mentaux, comme en témoigne l'augmentation du nombre de ces troubles dans les différentes révisions du célèbre Diagnostic and statistical manual of mental disorders (DSM), manuel de référence des praticiens et des chercheurs américains, et de plus en plus ailleurs dans le monde. Depuis la première version, éditée en 1952, on a multiplié par sept le nombre de troubles, passant de 60 à 410 dans la dernière version du DSM-IV (1994, révisé en 2000). Cette augmentation de nouvelles catégories de troubles, définies après 1987 comme d'origine biologique, accroît considérablement le nombre de personnes « concernées » et susceptibles de fournir un marché en pleine expansion pour l'industrie pharmaceutique. La dernière version du DSM-5, publiée en mai 2013, accentue cette tendance et suscite de nombreuses réactions dans plusieurs sections de l'Association américaine de psychologie. Dès 2010, Allen Frances - un des anciens coordonnateurs du DSM-IV - dénonçait l'augmentation considérable de nouveaux troubles mentaux par $1^{\circ}$ le biais de nouvelles catégories diagnostiques, qui concernent « [...] des manifestations extrêmement fréquentes en population générale » et $2^{\circ}$ «l'abaissement des seuils diagnostiques pour la plupart des troubles inclus dans le DSM-IV [...], ce qui aura pour effet de favoriser les traitements de masse utilisant des médicaments coûteux, à l'efficacité contestable et à l'innocuité mal établie ». Dans une interview parue dans Books, en avril 2013, 
il précise que le marché des troubles de l'attention est passé de 15 millions de dollars avant la publication du DSM-IV à 7 milliards aujourd'hui, soit un coefficient multiplicateur de 450 .

De la sorte, la pathologisation de processus normaux, liés aux processus de développement de l'individu, tout au long de sa vie (par exemple, les changements cognitifs et cérébraux, liés à l'avancée en âge, définis dans la $5^{\mathrm{e}}$ édition du manuel, comme des troubles neurocognitifs légers pour les personnes âgées [voir le blog Mythe Alzheimer, http://mythe-alzheimer.overblog.com] ou bien les troubles de la régulation de l'humeur chez l'enfant et l'adolescent) relève, là encore, d'une conception normalisatrice des conduites et d'une marche forcée vers la médicalisation des comportements. Comme le dénonce la pétition lancée par la Society for humanistic psychology, Division 32 de l'Association américaine de psychologie, ces nouveaux troubles n'ont aucune base scientifique solide issue des recherches cliniques et peuvent même être la conséquence de traitements inappropriés par neuroleptiques («Open letter to the DSM-5 », www.ipetitions.com/petition/dsm5/).

\section{CONCLUSION}

Dans un système d'économie de marché, il est urgent d'interroger la façon $1^{\circ}$ dont les connaissances en psychologie sur l'activité humaine (affective, sociale, cognitive) sont dévoyées au service de modèles de société marchands et individualistes, dans lesquels le psychologisme est un rempart indispensable à toute organisation collective de résistance et de forces créatives $; 2^{\circ}$ dont les technologies, notamment de neuro-imagerie, sont utilisées dans un but de normalisation des conduites et de naturalisation de $1^{\prime}$ esprit $; 3^{\circ}$ dont une conception strictement bio-médicale des parcours de vie (de l'enfance à la vieillesse) envahit peu à peu les domaines de recherche et les champs d'interventions en psychologie. 
Toutes ces questions de société doivent interpeller les «professionnels de la psychologie », chercheurs ou praticiens, au risque, s'ils ne s'en emparent pas, de cautionner l'idéologie libérale marchande et de participer à un système qui réduit connaissances et individus à des marchandises comme les autres.

\section{RÉFÉRENCES}

Bernays (Edward).- Propaganda, New York, H. Liveright, 1928.

Frances (Allen).- À propos des 19 «propositions » du DSM-5 : on ouvre la boîte de Pandore !, La lettre de psychiatrie française, 194, 2010, p. 10-14.

Gazzaniga (Michael S.).- Neuroscience and the correct level of explanation for understanding mind. An extraterrestrial roams through some neuroscience laboratories and concludes earthlings are grasping how best to understand the mind-brain interface, Trends in cognitive sciences, 14, 7, 2010, p. 291-292.

Gori (Roland).- La fabrique des imposteurs, Paris, Les liens qui libèrent, 2013.

Guillaume (Fabrice), Tiberghien (Guy), Baudouin (Jean-Yves).- Le cerveau n'est pas ce que vous pensez : images et mirages du cerveau, Grenoble, Presses universitaires de Grenoble, 2013.

Parazelli (Michel).- Les programmes positivistes de prévention précoce. Vers quel horizon politique ?, dans le collectif «Pas de 0 de conduite », Les enfants au carré ? Une prévention qui ne tourne pas rond!, Toulouse, Érès, 2011, p. 65-91.

Tiberghien (Guy).- Entre neurosciences et neurophilosophie : la psychologie cognitive et les sciences cognitives, Psychologie française, 52, 2007, p. 279-297. 
Tiberghien (Guy).- La neuro-imagerie cognitive : mythes et réalités, dans Pévet (P.), Sauvayre (R.), Tiberghien (G.), Les sciences cognitives : dépasser les frontières disciplinaires, Grenoble, Presses universitaires de Grenoble, 2011, p. 43-52.

Williams (Simon J.), Higgs (Paul), Katz (Stephen).- Neuroculture, active ageing and the older brain: Problems, promises and prospects, Sociology of health \& illness, 34, 2012, p. 6478.

Winkin (Yves).- La communication n'est pas une marchandise : résister à l'agenda de Bologne, Bruxelles, Labor, 2003. 
